\title{
Fairness aware resource allocation for downlink MISO-OFDMA systems
}

\author{
İlhan BAŞTÜRK \\ Electrical and Electronics Engineering Department, \\ Izmir Institute of Technology Urla, 35430, Izmir, Turkey \\ Email: ilhanbasturk@iyte.edu.tr
}

\author{
Berna ÖZBEK \\ Electrical and Electronics Engineering Department, \\ Izmir Institute of Technology Urla, 35430, Izmir, Turkey \\ Email: bernaozbek@iyte.edu.tr
}

\begin{abstract}
In this paper, a resource allocation problem for downlink multiple input-single output orthogonal frequency division multiple access (MISO-OFDMA) systems is investigated. The problem is defined as maximizing the minimum user rate with the constraints of total power and bit error rate (BER). Since it is difficult to obtain the optimal solution to this problem, a suboptimal but efficient solution is proposed based on zero forcing beamforming (ZFBF) to reduce computational complexity. The proposed algorithm is a fairness aware radio resource allocation algorithm that shares the resources equally among the users who has different distances from the Base Station (BS). The simulation results show that the proposed algorithm satisfies the fairness criterion having higher data rates compared to the existing algorithms.
\end{abstract}

\section{INTRODUCTION}

Orthogonal Frequency-Division Multiple-Access (OFDMA) which arises from Orthogonal Frequency Division Multiplexing (OFDM) and inherits of its superiority of mitigating multipath fading, is capable of providing higher spectral efficiency by exploiting inherent multiuser diversity. It is a promising candidate for future wireless communication systems that provides higher data rates by ensuring the Quality of Service (QoS) demands.

Multiple-Input Multiple-Output (MIMO) technology offers significant increase in the data throughput without additional bandwidth or transmit power requirements. OFDMA combined with MIMO increase the system capacity and improve reliability, but also introduces new problems relating to how these frequency and space subchannels are efficiently divided among users. For MIMO-OFDMA systems, adaptive resource allocation in frequency, time, and space is complex due to the large number of degrees of freedom to be handled. In recent years, many dynamic resource (subcarrier, power, and bit) allocation algorithms for single input-single output-orthogonal frequency division multiple access (SISO-OFDMA) systems have been developed to find the solution of maximizing channel capacity or minimizing the overall transmit power [1]. However, these algorithms cannot directly be applied to the MIMO-OFDMA systems. Thus, it is a necessity to develop new and effective resource allocation algorithms for these systems.

The resource allocation algorithms for MIMO-OFDMA systems can be classified as rate maximization or power minimization as in the SISO-OFDMA systems. Moreover, it can also be examined in two different scenarios. In the first scenario, subcarrier sharing is not allowed among users so each subcarrier is allocated to only one user in the cell [2]-[5]. This scenario makes the problem easier and co-channel interference is eliminated. In the second scenario, subcarrier sharing is allowed among users by allocating the spatial domain to different users at the same time and frequency [6]-[9]. Although, cochannel interference occurs, this scenario increases the system capacity because of the rising number of served users [10]. In this scenario, total number of users in a cell can be less or more than number of transmit antennas. The case, which includes the number of users is more than the number of transmit antennas, is more realistic for next cellular communication systems. Here, only a selected subset of all existing users is scheduled on each subcarrier. Co-channel interference, which is the main problem of the scenarios that allow subcarrier sharing, can be eliminated using different techniques such as dirty paper coding (DPC), block diagonalization (BD) and beamforming $(\mathrm{BF})$. DPC is a theoretically optimal technique and achieves maximum sum capacity, but it is proven to be difficult to implement in practice [11]. Beamforming (BF) is a suboptimal strategy that can also serve multiple users at a time, but with reduced complexity. Zero-forcing beamforming (ZFBF) is the simplest case of beamforming strategy where the weight vectors are chosen to avoid interference among user streams.

While designing resource allocation algorithms, it is important that all users in the cell fullfill their QoS requirements. The fairness must be provided among users by preventing the users, which have good channel conditions, to obtain most of the system resources. Fairness implies that all users get an equitable amount of system resources and can be viewed as a tradeoff between maximizing the system throughput and maximizing the equality between users. Most of the resource allocation algorithms in the literature ignores the fairness criteria and allow the users with good channel gains to obtain most part of the resources. In [6]-[7], suboptimal user selection and beamforming algorithms based on ZF have been proposed to maximize the system capacity without guaranteeing any kind of fairness among the users' data rates. The maximization of the data rate with the constraints of total available power and Bit Error Rate (BER) has been aimed in [8] while supporting a kind of fairness among users. In [9], a resource allocation 
algorithm has been developed that incorporates fairness by imposing proportional constraints.

In this paper, we propose a fairness aware resource allocation algorithm for downlink MISO-OFDMA systems. Our goal is to allocate system resources equally among users regardless of their distances from the BS by taking into account the BER requirements of all users' for the practically important case wherein the number of downlink users is larger than the number of transmit antennas, which entails user selection. Simulation results indicate that the proposed algorithm is not only satisfies the fairness criterion but also increase the minimum user capacity and total system capacity compared to the existing algorithms.

This paper is organized as follows. First, we describe the MISO-OFDMA system model and problem formulation in section II. Then, the proposed fair resource allocation algorithm is described in section III. Finally, we give the simulation results and conclusion in section IV and V respectively.

\section{System Model AND Problem Formulation}

A downlink MISO-OFDMA system model with $N$ subcarriers, $N_{t}$ transmit antennas at the BS and $K$ mobile users equipped with a single receive antenna is considered as shown in Figure 1. In this model, the number of total users is more than the number of transmitting antennas $\left(K>N_{t}\right)$, thus it is required to select $l \leq N_{t}$ users out of $K$ users in each subcarrier. The number of simultaneously served users on each subcarrier is limited by the number of transmit antennas. A perfect channel state information (CSI) is assumed at the transmitter, which means that each user estimates perfectly its wireless channel and feedback instantly the BS through an error-free feedback channel. There are $I=\sum_{l=1}^{N_{t}}\left(\begin{array}{c}K \\ l\end{array}\right)$ possible combinations of users transmitting on the same subcarrier denoted as $\xi_{i}$, where $\xi_{i} \subset\{1,2, \ldots, K\}, 0<\left|\xi_{i}\right| \leq N_{t}$, denotes the cardinality of set $\xi_{i}$.

When it is assumed that the user set selected on subcarrier $n$ is $\xi_{i}$, and user $k \in \xi_{i}$, the received signal of user $k$ on subcarrier $n$ is

$$
y_{n, k}=\mathbf{h}_{n, k} \sum_{j \in \xi_{i}} \sqrt{p_{n, j}} \mathbf{w}_{n, j} s_{n, j}+z_{n, k}
$$

where $s_{n, j}$ is the corresponding transmit symbol on subcarrier $n, \mathbf{h}_{n, k}$ is $1 \times N_{t}$ fading channel vector between the BS and user $k$ on subcarrier $n, \mathbf{w}_{n, j}$ is $N_{t} \times 1$ beamforming vector for user $j$ and $z_{n, k}$ is the Additive White Gaussian Noise (AWGN) with variance $\sigma_{n}^{2}$. Moreover, $p_{n, j}$ is the allocated power for subcarrier $n$. It is assumed that the total power is allocated equally among all subcarriers.

In order to cancel the interuser interference on the same subcarrier and provide spatial multiplexing, beamforming vectors are formed using $\mathrm{ZFBF}$ technique and the beams are steered into preferred directions of the selected users. First of all, the channel matrix of subset $\xi_{i}$ on subcarrier

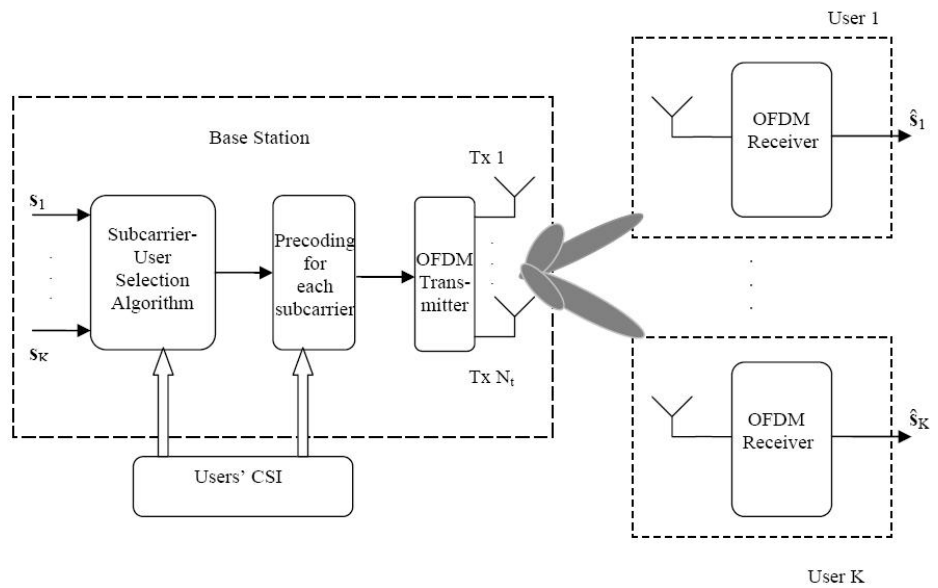

Fig. 1: MISO-OFDMA System Model.

$n, \quad \mathbf{H}_{n}\left(\xi_{i}\right)=\left[\mathbf{h}_{n, 1}^{T}, \ldots, \mathbf{h}_{n, N_{t}}^{T}\right]^{T}$, is obtained. Then, MoorePenrose pseudo-inverse of $\mathbf{H}_{n}\left(\xi_{i}\right)$ gives us the beamforming matrix $\tilde{\mathbf{W}}_{n}\left(\xi_{i}\right)=\left[\tilde{\mathbf{w}}_{n, 1}, \ldots, \tilde{\mathbf{w}}_{n, N_{t}}\right]$ which contains beamforming vectors belong to each user on subcarrier $n$. The pseudoinverse is given by

$$
\tilde{\mathbf{W}}_{n}\left(\xi_{i}\right)=\mathbf{H}_{n}\left(\xi_{i}\right)^{H}\left(\mathbf{H}_{n}\left(\xi_{i}\right) \mathbf{H}_{n}\left(\xi_{i}\right)^{H}\right)^{-1}
$$

To account for the power constraint on the precoding vectors, the vectors $\tilde{\mathbf{w}}_{n, i}$ are normalized by

$$
\mathbf{w}_{n, k}=\sqrt{\frac{\left|\xi_{i}\right|}{\operatorname{tr}\left\{\tilde{\mathbf{W}}_{n}\left(\xi_{i}\right) \tilde{\mathbf{W}}_{n}\left(\xi_{i}\right)^{H}\right\}}} \tilde{\mathbf{w}}_{n, k}
$$

As a result, $\mathbf{w}_{n, j}$ is an orthogonal basis for the null space of a matrix with vector $\mathbf{h}_{n, k}\left(j \neq k, k, j \in \xi_{i}\right)$ as its rows. Interuser interference on the same subcarrier is eliminated since the condition $\mathbf{h}_{n, k} \mathbf{w}_{n, j}=0$ is satisfied. Thus, the received signal becomes,

$$
y_{n, k}=\sqrt{p_{n, k}} \mathbf{h}_{n, k} \mathbf{w}_{n, k} s_{n, k}+z_{n, k} .
$$

M-QAM modulation is applied with a BER requirement, and the data rate for user $k$ on the subcarrier $n$ can be expressed as

$$
r_{n, k}=\log _{2}\left(1+p_{n, k} \gamma_{n, k}\right)
$$

where $\gamma_{n, k}=\left|\mathbf{h}_{n, k} \mathbf{w}_{n, k}\right|^{2} /\left(\sigma_{n}^{2} \Gamma\right)$ is the equivalent signal to noise ratio and $\Gamma=-\ln \left(5 B E R_{k}\right) / 1.5$ [14] and $B E R_{k}$ denotes the BER requirement of user $k$. The data rate of user $k$ can be written as

$$
R_{k}=\sum_{n=1}^{N} \sum_{i=1}^{I} \rho_{n, i, k} \log _{2}\left(1+p_{n, i, k} \gamma_{n, i, k}\right)
$$

where $\rho_{n, i, k}$ is the subcarrier allocation indicator that $\rho_{n, i, k}=$ 1 when the subcarrier $n$ is allocated to the user $k \in \xi_{i}$; otherwise $\rho_{n, i, k}=0$. 
Letting $B E R_{k}^{\text {target }}$ denote the target BER of user $k$, the optimization problem can be formulated as

$$
\max \min _{k} R_{k}
$$

subject to

$$
\begin{gathered}
p_{n, i, k} \geq 0, \forall n, i, k \\
\sum_{k=1}^{K} p_{n, i, k} \leq \frac{P_{\text {Total }}}{N}, \forall n, i \\
B E R_{k} \leq B E R_{k}^{\text {target }}, k=1, \ldots, K \\
\sum_{k=1}^{K} \rho_{n, i, k} \leq N_{t}, \forall n, i \\
\rho_{n, i, k} \in\{0,1\}, \forall n, i, k
\end{gathered}
$$

where $P_{\text {Total }}$ is the total transmitted power.

The optimal solution can be obtained by exhaustive search of all possible user assignment sets in all subcarriers but it has very high computational complexity. Thus, an efficient suboptimum solution is proposed in the next section.

\section{The Proposed Fair Resource Allocation}

Generally, users on the edge of cells or in deep fades can not obtain any resources so they can not be able to meet their QoS requirements. When the BS is determining the resource allocation for a given time slot, the notion of fairness plays a key role. Fairness implies that all users get an equitable amount of system resources. In this study, a fair resource allocation algorithm is proposed in order not to victimize the users far from the base station.

The proposed algorithm is given below in detail.

Step 1.

$\Lambda=1,2, \ldots, N, \quad n \in \Lambda, \quad R_{k}=0 \quad k=1,2, \ldots, K$

Step 2.

While $|\Lambda| \neq \emptyset$ do

* Set $\Psi_{0}=\{1, \ldots, K\}$ and $a=1$

* Find user $k^{\prime}$ satisfying, $k^{\prime}=\arg \min _{k} R_{k}$

* Find subcarrier $n$, which has the best channel gain with selected user,

$n=\arg \max _{j \in \Lambda}\left\|\mathbf{h}_{k^{\prime}, j}\right\|$

* Set $S_{a}=\left\{k^{\prime}\right\}, \Psi_{a} \leftarrow \Psi_{0} \backslash\left\{k^{\prime}\right\}, \mathbf{H}\left(S_{a}\right)=\mathbf{h}_{k^{\prime}, n}$

* Compute the achievable rate of the selected user $R\left(S_{a}\right)$.

* while $a \leq N_{t}$ do

- Increase a by 1

- $\mathbf{P}_{a}^{\perp}=\mathbf{I}_{N_{t}}-\mathbf{H}\left(S_{a-1}\right)^{H}\left(\mathbf{H}\left(S_{a-1}\right) \mathbf{H}\left(S_{a-1}\right)^{H}\right)^{-1} \mathbf{H}\left(S_{a-1}\right)$, where $\mathbf{I}_{N_{t}}$ is the $N_{t} \times N_{t}$ identity matrix, $\mathbf{H}\left(S_{a-1}\right)$ denotes the matrix consisting of the channel vectors of the users already selected in the first $a-1$ steps

- for $k \in \Psi_{a-1}$ do

$$
p_{k}=\left|\mathbf{h}_{k, n} \mathbf{P}_{a}^{\perp}\right|^{2}=\mathbf{h}_{k, n} \mathbf{P}_{a}^{\perp} \mathbf{h}_{k, n}^{H}
$$

end for

- Form a group, $\zeta$, of candidates that contains the $M$ users with the largest values of $p_{k}, k \in \Psi_{a-1}$

- Compute $r_{m, n},(m=1, . ., M)$ by using Equation (5), which is the data rate of each user in $\zeta$, if they are allocated to subcarrier $n$.

- Find user $k^{*}$ satisfying, $k^{*}=\arg \max _{m} \frac{r_{m, n}}{R_{m}(t-1)+r_{m, n}}, k^{*} \in \zeta$

- Compute the achievable rate $R\left(S_{a-1} \cup\left\{k^{*}\right\}\right)$ in order to decide to admit this user to subcarrier $n$

- If $R\left(S_{a-1} \cup\left\{k^{*}\right\}\right) \geq R\left(S_{a-1}\right)$ do $S_{a}=S_{a-1} \cup\left\{k^{*}\right\}, R\left(S_{a}\right)=R\left(S_{a-1} \cup\left\{k^{*}\right\}\right)$ $\Psi_{a} \leftarrow \Psi_{a-1} \backslash\left\{k^{*}\right\}$,

$\mathbf{H}\left(S_{a}\right)=\left[\mathbf{H}\left(S_{a-1}\right)^{T} \mathbf{h}_{k^{*}, n}^{T}\right]^{T}$.

- Else do

Set $a=N_{t}+1$ to terminate the allocation loop in $n$. end if

\section{end while}

* Update $R_{k}$ according to (5)-(6), $\forall k \in S_{a}$

$* \Lambda \leftarrow \Lambda \backslash\{n\}$

\section{end while}

In the proposed algorithm outlined above, $\Psi$ and $\Lambda$ represents the user set and subcarrier set, respectively. First step of the algorithm is the initialization part in which the rate values of each user is set to zero.

Maximum $N_{t}$ users are selected for each subcarrier in the second step of the algorithm. The important point of this selection procedure is to provide fairness without decreasing the system capacity too much while allocating the system resources. In our algorithm, the subcarrier selection priority is given to the user which has the minimum data rate. During the first iteration, when data rates of all users are zero, random initialization is performed, in other words, any user is chosen arbitrarily. Then, subcarrier which has the best channel gain with that selected user is determined. So, a user-subcarrier pair is obtained. Later, the users which are orthogonal to the already selected users and increase the sum data rate are admitted for that subcarrier. Each remaining channel vectors $\mathbf{h}_{k, n}, k \in \Psi_{a-1}$ belongs to users not selected yet, is projected onto the orthogonal complement of the subspace spanned by the channels of the selected users by calculating the projector matrix, $\mathbf{P}^{\perp}$ [12].

A user group, $\zeta$, with a size $M=\min \left\{\Psi_{a-1}, N_{t}\right\}$, which is decided heuristically, is formed. After that, the data rates of the users, $r_{m, n}$, in $\zeta$ are calculated assuming that they are admitted to the subcarrier $n$. At a decision time, $t$, if the subcarrier $n$ is allocated to user $m$, then user $m$ 's total data rate is updated by $R_{m}(t)=R_{m}(t-1)+r_{m, n}$, where $R_{m}(t-1)$ is user $m$ 's data rate at time $(t-1)$ [8]. By using these equations, the user that maximizes the proportional fairness value, $\frac{r_{m, n}}{R_{m}(t-1)+r_{m, n}}$ is selected for that subcarrier. This implies that the user with better channel condition takes higher priority in resource allocation. However, once user $m$ has been selected on subcarrier $n, R_{m}$ will be larger and the chance 
of selecting another subcarrier will be decreased at the next decision time $(t-1)$. After selecting the user which has a maximum proportional fairness value, it is controlled if it is increasing the sum rate of this subcarrier in order to admit this user to subcarrier $n$. After allocating all users for the subcarrier $n$, the data rates of the users belong to this subcarrier are updated using Equations (5)-(6) and the algorithm is repeated until the subcarrier set $\Lambda$, is empty.

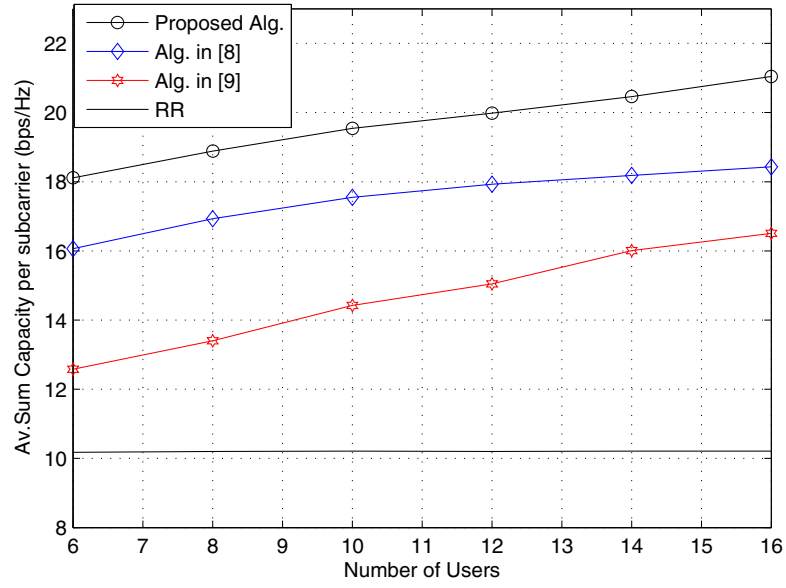

Fig. 2: Average sum data rate vs number of users.

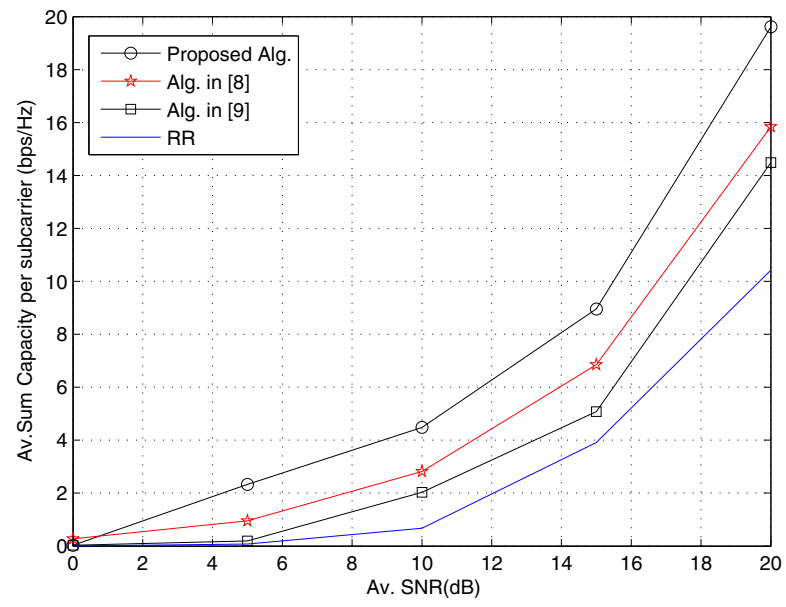

Fig. 3: Average sum data rate vs SNR

\section{Simulation Results}

We compare the proposed algorithm with the existing ones presented in [8][9] and Round-Robin (RR) algorithm. In RR algorithm each user is given a fair share of the channel resource regardless of the channel state and $N_{t}$ users are selected in each subcarrier.

In the simulations, BS which is in the centre of a single cell system has $N_{t}=4$ transmitting antennas and all users

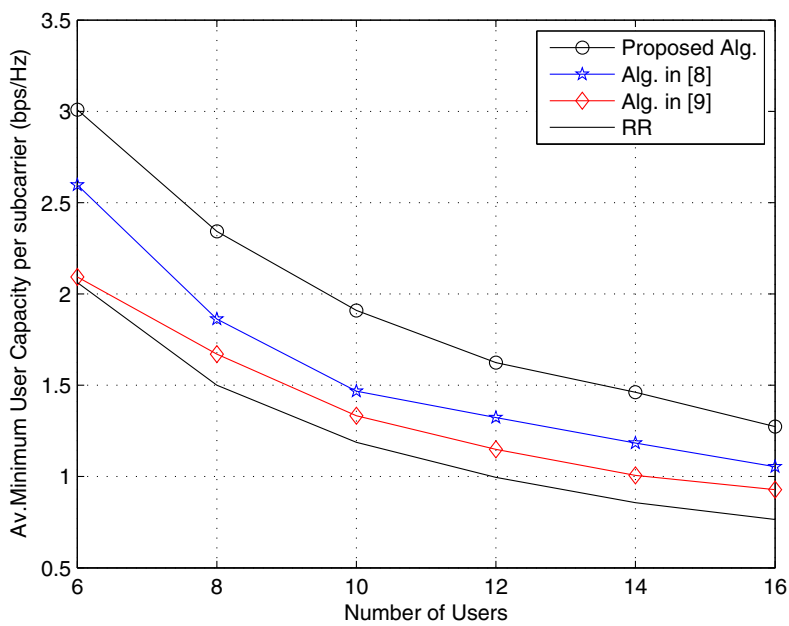

Fig. 4: Minimum user Rate per subcarrier vs number of users.

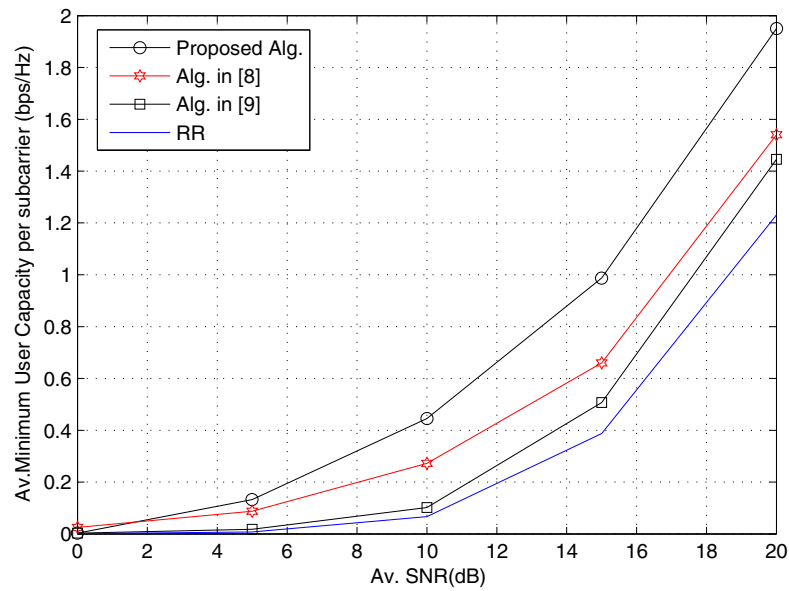

Fig. 5: Minimum user Rate per subcarrier vs SNR

around the BS have single receive antenna. The number of subcarriers is set to $N=64$. The time varying frequency fading channel has six taps and the Doppler shift is chosen to be $100 \mathrm{~Hz}$. Each taps is Rayleigh distributed and the conventional exponential decay multipath channel model is used for power-delay profile. Since the users' distances from the BS are different for each user, pathloss is also considered and $L=28.6+35 \log 10(d)(\mathrm{dB})$ is used as pathloss model. Proportional data rate constraints in [9] are set to one.

We calculate the sum data rate per subcarrier using the Equations (5)-(6) for different number of users and different SNR values as shown in Figure 2 and Figure 3, respectively. It is assumed that the target BER of each user is $10^{-3}$. In Figure 2, SNR value is set to $20 d B$ and in Figure 3, the results are obtained for $K=10$ users. It is seen that the proposed algorithm gives the highest sum rate performances.

Figure 4 and 5 show that the minimum user data rate per 


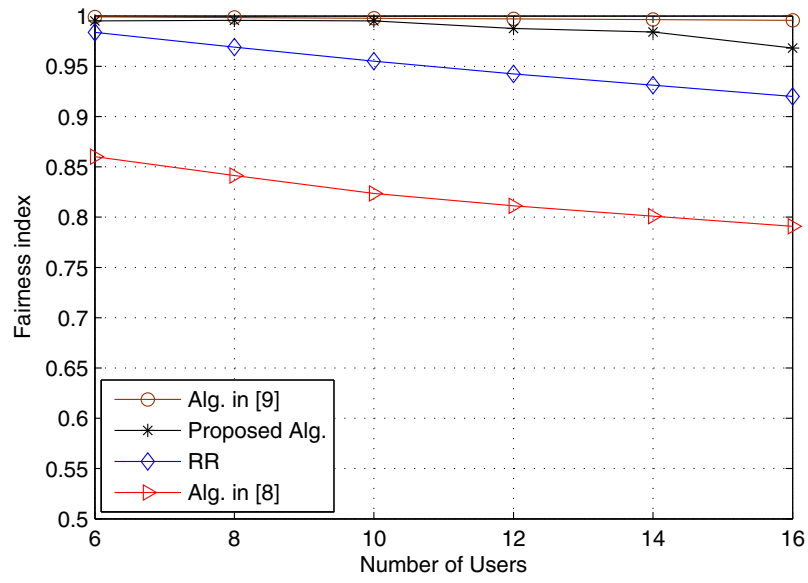

Fig. 6: Fairness Index.

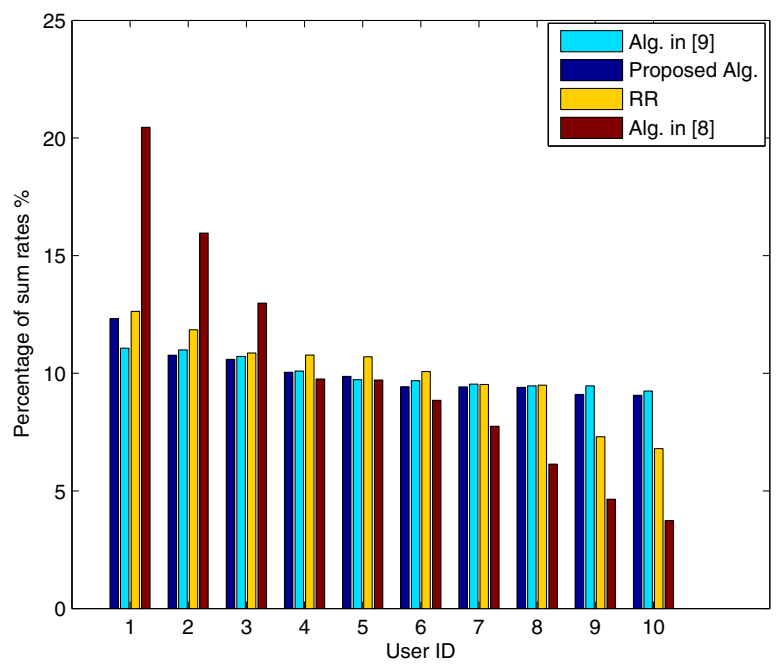

Fig. 7: Percentage of sum data rates among users.

subcarrier for the different number of users and different SNR values, respectively. Also, SNR value is set to $20 d B$ in Figure 4 , and the number of users $K$, is selected 10 in Figure 5. It is revealed that the proposed algorithm maximizes significanlty the minimum user data rate.

Fairnes index performances which show allocation of the resources equally among the users are given in Figure 6 . The fairness index is calculated by $F I=\frac{\left(\sum_{k=1}^{K} R_{k}\right)^{2}}{K \sum_{k=1}^{K}\left(R_{k}\right)^{2}}$ which is introduced in [13]. As seen in the figure that our proposed algorithm satisfies the fairness criterion. It almost allocates the system resources equally. Moreover, Figure 7 compares the fairness of the algorithms. In this figure, the percentage indicating how the sum data rate is allocated among users are given when the number of users $K$ is 10 and $S N R$ is $15 d B$. The users are labeled by their path losses; users closer to the BS have smaller IDs. The users with smaller IDs have good channel conditions and get most of the resources in RR and the algorithm in [8]. The algorithm in [9] is the most fair among all and the proposed algorithm has approximately equal fairness among all users.

\section{CONCLUSION}

In this paper, we have proposed a fairness aware resource allocation algorithm for downlink MISO-OFDMA systems. The proposed algorithm is based on zero-forcing beamforming. We compared the proposed algorithm with existing resource allocation algorithms in terms of fairness index and capacity. The proposed algorithm provided a balance between fairness and system capacity of the system. The minimum user data rate is maximized and the available capacity is distributed fairly among all users. Therefore, the users that have poor channels are not aggrieved.

\section{REFERENCES}

[1] D. Le Ruyet, B. Ozbek, "Resource Management Techniques for OFDMA" , book chapter in Orthogonal Frequency Division Multiple Access Fundamentals and Applications, Auerbach Publications, pp.101-129, 2010

[2] J. Xu, J. Kim, W. Paik, and J.-S. Seo, "Adaptive Resource Allocation Algorithm with Fairness for MIMO-OFDMA System," in Proc. VTC 2006-Spring Vehicular Technology Conference IEEE 63rd, vol. 4, 2006, pp. 1585-1589.

[3] S. Qiaoyun, T. Hui, D. Kun, W. Shengdong, and Z. Ping, "A novel resource allocation algorithm for multiuser downlink MIMO-OFDMA," in IEEE Wireless Communications and Networking Conference, WCNC 2008, pp. 1844-1848.

[4] Bin Da, Chi Chung Ko, "Resource Allocation in Downlink MIMOOFDMA with Proportional Fairness", Journal of Communications, Vol 4, No 1 (2009), pp.8-13.

[5] Mraz A., Zambo T., Imre S.,"Radio Resource Management for MIMOOFDMA Access in Rayleigh Fading Channel.", European Wireless Conference, pp.261-268, 2010

[6] Petermann M., Bockelmann C., Kammeyer, K. D.,"On Allocation Strategies for Dynamic MIMO-OFDMA with Multi-User Beamforming",12th International OFDM-Workshop, Hamburg, Germany, 2007.

[7] Zhong C., Li C., Zhao R., Yang L., Gao X., "Dynamic Resource Allocation for Downlink Multi-user MIMO-OFDMA/SDMA Systems", IEEE ICC'09,Dresden, Germany, June 2009

[8] S. Kai, W. Ying, C. Zi-xiong, Z. Ping, " Fairness based resource allocation for multiuser MISO-OFDMA systems with beamforming", J. China Univ. of Posts and Telec., vol 16, n. 1, pp.38-43, 2009.

[9] Papoutsis, V.D., Fraimis, I.G., Kotsopoulos, S.A.,"User selection and resource allocation algorithm with fairness in MISO-OFDMA", IEEE Communications Letters, vol 14, n.5, pp. 411-413, May 2010

[10] Q. H. Spencer, A.L. Swindlehurst, M. Haardt, " Zero-forcing methods for downlink spatial multiplexing in multi-user MIMO channels", IEEE Trans. Sig. Proces. vol 52, n.2, pp.461-471, 2004.

[11] Costa, M. (1983) “Writing on dirty paper", IEEE Trans. on Information Theory, Vol. 29, No. 3, pp.439-441.

[12] Z. Tu, R. S. Blum, "Multiuser diversity for a dirty paper approach", IEEE Commun. Lett., vol 7, n.8, pp.370-372, 2003.

[13] R. Jain, D.M. Chiu, W.R. Hawe, "A Quantitative Measure of Fairness and Discrimination for Resource Allocation Shared Computer Systems", Digital Equipment Corporation technical report TR-301, 1984.

[14] Goldsmith A J, Chua S."Variable-rate variable-power MQAM for fading channels". IEEE Transactions on Communications, 45(10): pp.12181230,1997 